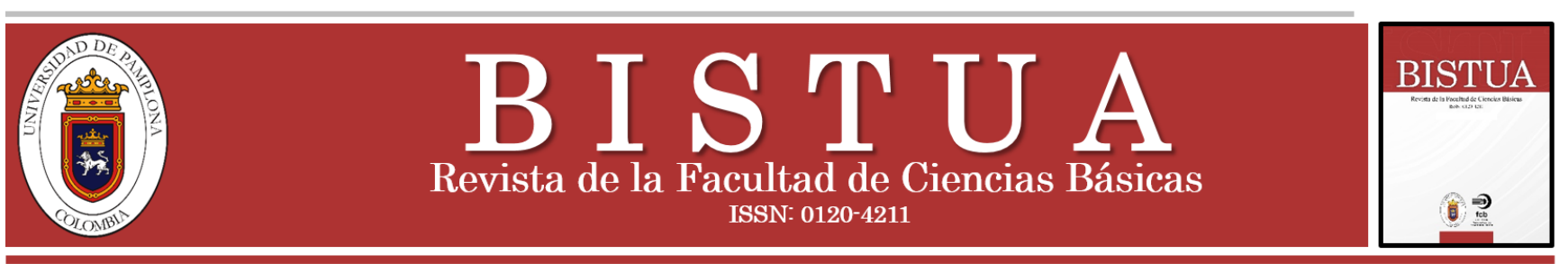

http://revistas.unipamplona.edu.co/ojs_viceinves/index.php/BISTUA

\title{
Harina de grillo Acheta domesticus: composición lipídica y posibilidades sobre su modificación por medio de la dieta
}

\section{Acheta domesticus cricket flour: lipid composition and possibilities of dietary modification}

\author{
Pol-Jon Álvarez ${ }^{a}$; Javier Mateo ${ }^{a} ;$ Javier Giráldez ${ }^{b}$ \\ ${ }^{a}$ Departamento de Higiene y Tecnología de los Alimentos, C.P. 24008, España. \\ ${ }^{b}$ Instituto de Ganadería de Montaña, Consejo Superior de Investigaciones Científicas, C.P. 24346, España. \\ Contacto: palvam06@estudiantes.unileon.es
}

Recibido: Noviembre 6, 2020. Aceptado: Diciembre 27, 2020

https://doi.org/10.24054/01204211.v2.n2.2020.4442

\section{Resumen}

Actualmente la producción de insectos como alimento no étnico está incrementándose y podría convertirse en una producción intensiva de importancia en un futuro no muy lejano. La especie de grillo Acheta domesticus presenta una anatomía y una fisiología favorables para su producción en intensivo. Además, su harina tiene alto valor nutritivo, especialmente reconocido por su elevado contenido en proteína y minerales. Hay una creciente cantidad de estudios sobre la producción de este grillo y la composición de su harina, que demuestran el interés científico que suscita. Esta harina es una gran fuente de proteínas explotable en alimentación humana y animal, pero su contenido lipídico es también de gran importancia. La cantidad de grasa de la harina de grillo está entorno al 10-16\% (sobre materia seca) y su perfil lipídico presenta aspectos muy favorables desde el punto de vista de la nutrición humana, dado que la mayoría de los ácidos grasos son insaturados, siendo mayoritarios los poliinsaturados. Ésta es una diferencia notable con la carne de las especies animales de abasto, las cuales representan una fuente de proteína habitual en alimentación humana, pero cuyos perfiles lipídicos contienen unos niveles muy inferiores de ácidos grasos poliinsaturados. Es remarcable también la posible modificación de la cantidad de grasa y el perfil de ácidos grasos de la harina de grillo mediante cambios en su dieta. Aún quedan muchos ámbitos que estudiar en profundidad para optimizar la producción de estos insectos, cuyo potencial para su empleo en la industria alimentaria es progresivo.

Palabras clave: Acheta domesticus; lípidos; producción insectos; perfil lipídico; modificación lipídica; harina de insectos.

\section{Introducción}

La ingesta de insectos, sin duda, ha tenido que ver en la evolución de las especies terrestres situadas en las partes más

\section{Abstract}

Nowadays, insect production as non-ethnic food is increasingly growing, and it appears that in a near future intensive insect production would become significant. The species of Acheta domesticus presents a favorable anatomy and physiology for intensive production. In addition, the cricket flour has a high nutritional value, especially recognized for its high protein and mineral contents. There is an increasing amount of scientific literature on the production and composition of this species that shows the scientific interest that it arouses. This flour is a great source of exploitable proteins for human and animal nutrition, but his lipid content has as well a great importance. The amount of fat in the cricket's flour is around 10-16\% (on dry matter basis), and from a nutritive point of view, its lipid profile presents positive aspects since most of its fatty acids are unsaturated, being the majority polyunsaturated. This is a notable difference with the meat from other livestock species, which represent a main source of protein in human nutrition, but whose lipid profiles contain much lower levels of polyunsaturated fatty acids. It is also remarkable the possibility of modifying the amount and lipid profile in cricket flour through dietary manipulation. There are still many subjects to study in deep for optimizing the production of these insects, whose potential in the food industry is rising progressively.

Keywords: Acheta domesticus; lipids; insect production; lipid profile; lipid modification; insect flour formatting. altas de la cadena trófica, por haber sido y ser parte de la dieta de multitud de estas especies [1]. Los humanos formamos parte de dicho grupo. Actualmente, coexisten culturas en las que se ha mantenido de forma tradicional la entomofagia y otras en las que 
no, pero lo que es innegable es la cantidad de posibilidades que brinda la explotación de insectos para alimentación tanto animal como humana. Su producción está en crecimiento e instituciones como la FAO promueven este tipo de explotaciones [2].

La producción industrial o semi-industrial de la especie de grillo Acheta domesticus para la obtención de harina destinada a la alimentación animal o humana presenta ventajas conocidas, como un gran contenido en proteínas, superior al $70 \%$, que además son de gran valor biológico debido a sus buenos índices de aminoácidos esenciales (EAAI), con un valor medio de $74,7 \%$. Este valor de EAAI es algo inferior al de la proteína de ave (78\%) o de pescado (82\%), pero superior al que presentan las proteínas vegetales, como el arroz (72\%) o algunas legumbres (68\%) [3]. Otro factor de gran interés es la gran versatilidad de esta especie para adaptarse a diferentes tipos de alimentación, lo que brinda la posibilidad de utilizar desechos y subproductos de la industria agroalimentaria para su alimentación [4]. Esta capacidad de adaptación a diversas dietas es especialmente reseñable a la hora de evaluar cómo a través de éstas se puede modificar la composición de la harina producida.

Como características generales de interés de esta especie cabe mencionar que tardan aproximadamente 7 semanas en llegar a adultos y que necesitan unos parámetros ambientales de temperatura y humedad controlados para optimizar su crecimiento, aunque tienen buenas capacidades de adaptación. Las densidades para su supervivencia son muy rentables, de entre 6000 y 12000 grillos por $\mathrm{m}^{2}$ [5]. Por tanto, estos animales, tienen un interesante potencial en relación con la sostenibilidad de los sistemas alimentarios. Su producción puede tener una comparativamente baja huella ecológica y favorecer más que otras a la economía circular [2], cuyo concepto está cobrando gran importancia para hacer frente al cambio climático en los años venideros.

El valor dietético y nutricional de la harina de grillo ofrece muy buenos resultados [3], es una fuente de vitaminas, minerales y presenta propiedades antioxidantes. También presenta buenas propiedades tecnológicas para elaborar productos alimenticios, como es el caso de masas fermentadas [6]. Por consiguiente, su implementación en productos elaborados como harinas de panadería, repostería, barras proteicas o snacks producidos mediante extrusión podría resultar en un aumento paulatino de su uso en alimentación humana. Además, debido a sus características podrían incluso administrarse como suplementos nutricionales para dietas específicas [7]. Su utilización también podría estar indicada para la producción de alimentos catalogados como bajos en grasa, o también en la elaboración de alimentos reconocidos como fuente de proteínas por tener valores superiores al 15\% [8]. En definitiva, la producción de Acheta domesticus se presenta como una opción con amplias posibilidades.

Actualmente la producción de grillo en intensivo aún está desarrollándose. Por ello cabe resaltar la necesidad de aumentar la investigación en la formulación de dietas para alimentar a estos animales, buscando optimizar la producción y aprovechar su potencial. Los insectos en general, forman parte de los llamados nuevos alimentos "Novel Food", siendo el grillo Acheta domesticus una de las especies de insecto más prometedoras de cara al futuro debido tanto a sus buenos resultados nutricionales, como a su bajo índice de transformación del alimento [9]. El objetivo de este estudio es esclarecer las oportunidades que brinda la implementación de la producción de estos animales de modo intensivo para consumo tanto humano como animal y vislumbrar las posibilidades de modificar el perfil lipídico de la harina producida.

\subsection{Metodología}

Para la realización de esta revisión se han consultado principalmente artículos científicos encontrados mediante los buscadores Google académico y ScienceDirect. El intervalo temporal que se tuvo en cuenta comprende desde 1950 hasta 2020. Los términos utilizados en el buscador para seleccionar los artículos han sido combinaciones de "Acheta domesticus" con los siguientes términos: "Nutritional value", "Edible insects", "Morphology", "House cricket farming", "Digestive enzymes", "Fatty acids", "Diet", "Omega 6 Omega 3" y "Cricket farming". También se utilizaron libros encontrados mediante el buscador Google Libros. Los términos utilizados fueron: "Acheta domesticus", "Edible insects", "Entomofagia" "Physiology insects", "Cricket farming", "Tablas composición". Se seleccionaron aquellos artículos relativos al efecto del metabolismo digestivo y el sistema de alimentación de los grillos sobre la composición de la harina, en concreto sobre el contenido y composición lipídica.

\section{Resultados}

\subsection{Metabolismo lipídico de los grillos y composición de la dieta para su cría en cautividad}

El sistema digestivo de la especie Acheta domesticus está dividido en 3 partes, estomodeo, mesodeo y proctodeo. Fisiológicamente en él tienen lugar múltiples reacciones. En el estomodeo comienza la digestión con la secreción de la enzima amilasa. En el mesodeo se lleva a cabo parte importante de la digestión con la secreción de glucosidasas y proteasas entre otras. En el proctodeo se secretan principalmente carbohidrasas y la digestión de los lípidos se lleva a cabo gracias a las lipasas [10]. Los principales procesos de absorción de los compuestos lipídicos se llevan a cabo por emulsión o resíntesis [11].

Los ácidos grasos linoleico y $\alpha$-linolénico se consideran, en general, como ácidos grasos esenciales para los animales. No obstante, la especie Acheta domesticus es capaz de sintetizar el ácido linoleico 18:2 ( $\omega-6)$, así como elongar y desaturar estos ácidos grasos hasta formar los ácidos grasos 20:3 y 20:4 ( $\omega-6)$, sin mediación de la microbiota digestiva. Para sintetizar el ácido linoleico usan una enzima en particular, la desaturasa $\Delta 12$, que poseen algunas especies de insectos, lo que les permite la síntesis del ácido linoleico "de novo" [12]. En el caso del grillo, se ha identificado que los mayores niveles de esta enzima se encuentran hacia las 39 
últimas mudas, antes de ser adultos. Esta es una enzima microsomal, en la que el NADPH funciona como cofactor y utiliza el oleoyl-CoA como sustrato (en algunos vegetales también existe, pero utiliza fosfolípidos como sustrato).

La composición de la dieta que se les administra ha sido objeto de estudio desde hace más de cincuenta años. Patton (1967) [13] comparó un total de 16 dietas oligídicas (a base de componentes orgánicos no purificados) diferentes. Este autor observó que las dietas que mejores resultados proporcionaron presentaban contenidos de proteína entre el $20 \%$ y $30 \%$, de carbohidratos entre el $32 \%$ y el $47 \%$ y de lípidos entre el 3,2\% y el 5,2\%. Como en la producción de otras especies, uno de los objetivos que se buscan es lograr que el pienso administrado tenga el mínimo coste [14]. Es debido a este interés que en el estudio realizado por Collavo et al. (2005) [4] se comprobó que esta especie de insecto es capaz de alimentarse de una amplia variedad de productos de desecho o subproductos de la industria de la agroalimentación, tanto humana como animal. En el estudio mencionado se comparaban los parámetros productivos obtenidos con cuatro dietas diferentes, siendo la dieta a base de subproductos de la industria agroalimentaria (restos, pieles de vegetales y frutas, arroz y pasta, carne de vacuno y porcino, pan y restos de quesos) la que mostró que, además de reducir el coste de la dieta, mejoraba, respecto a las otras dietas evaluadas, las tasas de crecimiento y disminuía la de mortalidad [4]. Este dato es de suma importancia, puesto que constata la viabilidad de utilizar subproductos en su alimentación de forma habitual. El impacto medioambiental de la producción de esta especie ya es considerablemente bajo en comparación con otras especies tanto a nivel de emisión de gases de efecto invernadero, como por la baja cantidad de agua que precisan [2]. Esta posibilidad permitiría ayudar a reducir el impacto medioambiental producido por el desperdicio de alimentos, sustentando así el desarrollo de una economía circular [15].

\subsection{Contenido en lípidos y perfil de ácidos grasos en la harina de grillo}

Los lípidos son un pilar importante a la hora de llevar una correcta nutrición y por ende tiene interés el disponer de estrategias en la producción animal para modificar tanto el contenido total de lípidos, como su perfil. El contenido medio de lípidos en la harina de grillo esta alrededor del 15\% [16]. Este contenido puede verse afectado sustancialmente por factores como el momento de desarrollo en el que se encuentre el animal [17], su sexo [3] o incluso por el método de sacrificio empleado, aunque éste último parece afectar en menor medida [18]. No obstante, el factor principal que puede afectar al tenor graso es la alimentación. En el artículo publicado recientemente por Bawa et al. (2020) [19] se puede observar que variando la dieta de los grillos se ha conseguido obtener un rango de contenido lipídico tan amplio como 8,943,9 g de lípidos/100 g materia seca. En el artículo se comenta que el hecho de que alguna de las dietas favorezca la formación de grasa (y el de proteínas descienda) se puede atribuir a un exceso de carbohidratos en la dieta. El razonamiento propuesto es que, si las proporciones de carbohidratos, grasa y proteína de la dieta no están ajustadas a las necesidades fisiológicas y necesidades físicas, la energía sobrante se almacenará como grasa. Esto sería una explicación de que los grillos alimentados con un nivel de proteína adecuado, pero con un contenido elevado de carbohidratos presenten mayores niveles de grasas.

En la Tabla 1 se presenta el perfil de ácidos grasos de harina de grillo de la especie Acheta domesticus observado por diferentes autores. Como se puede apreciar la mayor proporción corresponde a los ácidos grasos insaturados, con una media de $63 \%$, y dentro de estos, a los ácidos grasos poliinsaturados. Dentro de los ácidos grasos saturados, el ácido palmítico es el mayoritario (22\%). En lo que respecta a los insaturados, los ácidos mayoritarios son los ácidos linoleico $(36 \%)$ y oleico $(24 \%)$.

La grasa de grillo tiene un elevado interés nutricional. El ácido linoleico 18:2 ( $\omega-6)$ es un ácido graso esencial de gran interés en alimentación [16]. Como se ha comentado anteriormente, esta especie se desmarca significativamente de la mayoría de las especies de insecto por su capacidad para sintetizar ácido linoleico [20], lo que resulta en un alto contenido en el mismo (Tabla 1). Por otra parte, la ratio entre ácidos grasos saturados y poliinsaturados es un marcador de lo saludable que es una grasa comestible. Lo más recomendable es que la ratio en la grasa ingerida tenga un valor que cercano a 1 [21]. Como se puede apreciar la grasa de la harina de grillo cumple con dicho criterio.

Tabla 1. Contenido en ácidos grasos de la harina de grillo expresado en \%

\begin{tabular}{|c|c|c|c|c|}
\hline Ácido graso & $\begin{array}{c}\text { Tzampa- } \\
\text { Sosa et al. } \\
(2014) \\
{[22]}\end{array}$ & $\begin{array}{c}\text { Oonincx } \\
\text { et al. } \\
(2015) \\
{[23]}\end{array}$ & $\begin{array}{c}\text { Paul et } \\
\text { al. } \\
(2017) \\
{[16]}\end{array}$ & $\begin{array}{c}\text { Sánchez- } \\
\text { Muros et } \\
\text { al. (2020) } \\
{[17]}\end{array}$ \\
\hline C12:0 Láurico & 0,30 & 1 & 0,1 & - \\
\hline $\begin{array}{l}\text { C13:0 } \\
\text { Tridecanoico }\end{array}$ & - & 14,87 & - & - \\
\hline C14:0 Mirístico & 1,80 & 0,66 & 0,44 & 0,6 \\
\hline C16:0 Palmítico & 25,99 & 16,1 & 22,65 & 24,7 \\
\hline C16:1 Palmitoleico & 2,77 & 0,18 & 0,34 & 1,4 \\
\hline C18:0 Esteárico & 6,09 & 2,4 & 8,54 & 9,2 \\
\hline C18:1, 11-trans & 0,21 & 0,2 & - & - \\
\hline C18:1n9 Oleico & 29,14 & 21,34 & 20,18 & 24,4 \\
\hline C18:2n6 Linoleico & 29,11 & 38,52 & 41,39 & 36,3 \\
\hline $\begin{array}{l}\text { C18:3n3 } \\
\text { Linolénico }\end{array}$ & 1,56 & 2,35 & 1,11 & 1 \\
\hline
\end{tabular}




\begin{tabular}{lcccc}
$\begin{array}{l}\text { C23:0 } \\
\text { Tricosanoico }\end{array}$ & - & 0 & 0,02 & - \\
$\begin{array}{l}\text { Ácidos grasos } \\
\text { saturados }\end{array}$ & 34,18 & 35,03 & 31,73 & 34,5 \\
$\begin{array}{l}\text { Ácidos grasos } \\
\text { monoinsaturados }\end{array}$ & 31,91 & 21,52 & 20,52 & 25,8 \\
$\begin{array}{l}\text { Ácidos grasos } \\
\text { poliinsaturados }\end{array}$ & 30,67 & 40,87 & 42,5 & 37,3 \\
\hline
\end{tabular}

En la Figura 1 se presenta el valor medio del contenido de ácidos grasos saturados, monoinsaturados y poliinsaturados en la carne de ave, de cerdo, de vacuno, de cordero y en la harina de grillo. Como se puede apreciar, las carnes provenientes de animales de abasto (vertebrados) presentan, en general, proporciones muy bajas de ácidos grasos poliinsaturados (todas inferiores al 17\%), en comparación con la harina de grillo $(38,96 \%)$.

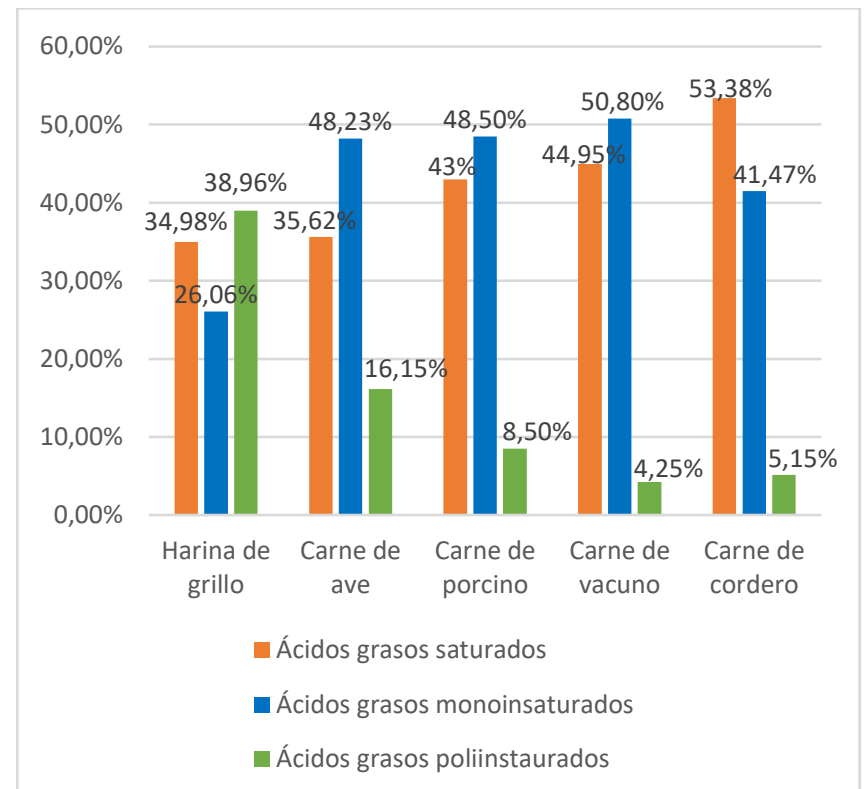

Figura 1. Comparativa de los porcentajes de ácidos grasos saturados, monoinsaturados y poliinsaturados entre la harina de grillo y la carne de distintas especies de animales de abasto. Adaptado de Moreiras et al. (2001) [24].

\subsection{Posibilidades de modificación del perfil lipídico de la harina de grillo}

El contenido en ácidos grasos poliinsaturados en la harina de grillo está fuertemente influenciado por el perfil lipídico de la dieta. Del estudio realizado por Collavo et al. (2005) [4] se deducen tres influencias principales. Primeramente, que, a mayor cantidad de ácidos grasos saturados suministrados en la ración, más ácidos grasos saturados se encuentran tanto en la harina como en heces. Por otra parte, aunque se suministren cantidades escasas de ácidos poliinsaturados en la dieta, se llegaría a niveles parecidos en la harina de grillo a aquellos alcanzados con valores de suministración de ácidos grasos poliinsaturados considerados normales. Por último, si se suministra gran cantidad de ácidos grasos poliinsaturados, el valor de estos aumenta también en la harina.

Desde el punto de vista de la salud humana, no solo es deseable un alto contenido en ácidos grasos poliinsaturados, sino lograr una relación $\omega-6 / \omega-3$ cercana a $1: 1$. Existen numerosas patologías en las cuales los valores de ácidos grasos $\omega-6$ y $\omega-3$ juegan un papel crucial [21]. Debido a cambios recientes en la dieta, la dieta normal del humano ha pasado de ratios 1:1 ó 2:1 a incluso 20:1 en algunos casos. Por este motivo se considera necesario promover el consumo de alimentos ricos en $\omega-3$, de manera a equilibrar la dieta [21].

En este sentido, Oonincx et al. (2020) [25] observaron que, al incrementar la proporción de aceite de lino (rico en ácido $\alpha$-linolénico, $(\omega-3)$ en la dieta de los grillos, se incrementó considerablemente el valor del ácido $\alpha$ linolénico, pasando de $0,8 \%$ (sin suplementación) a $12,7 \%$ con una suplementación de $4 \%$ de aceite de lino, tal y como refleja la Figura 2. Así pues, el contenido original se multiplicó por 16. Aparte de este aumento, según se iba incrementando el contenido de aceite de lino en la dieta, también se observó una disminución de las proporciones de ácidos grasos saturados y monoinsaturados.

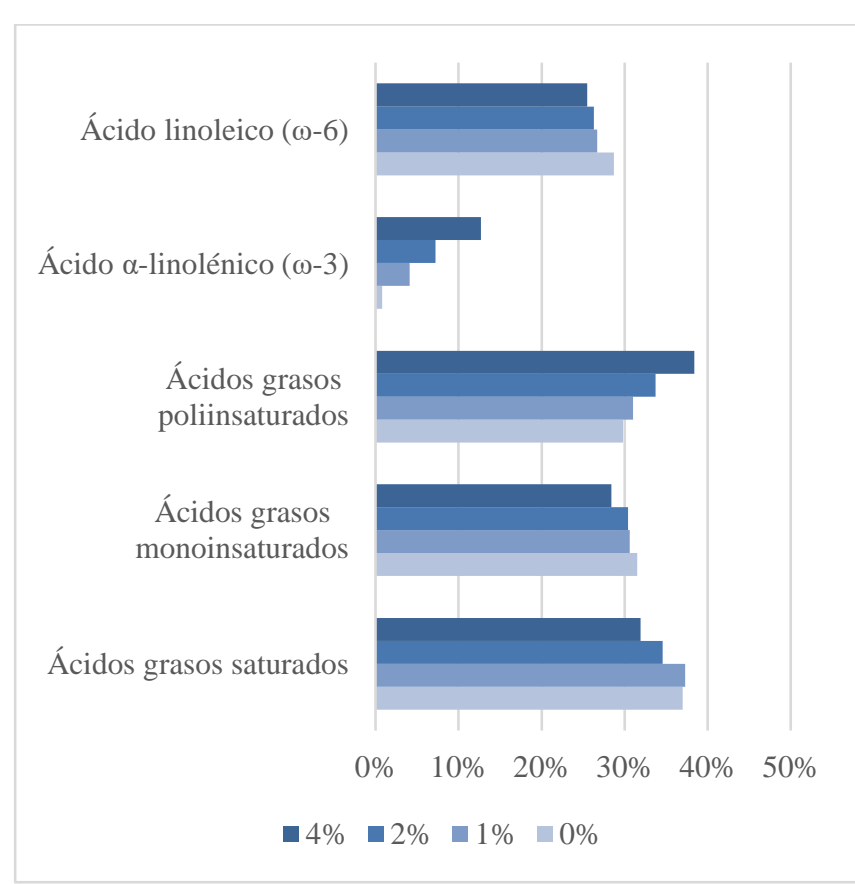

Figura 2. Cambios en los porcentajes de los ácidos grasos saturados, monoinsaturados, poliinsaturados, $\mathrm{n} 3(\omega-3)$ y $\mathrm{n} 6(\omega-6)$ en función del porcentaje de contenido de aceite de lino en la dieta. Adaptado de Oonincx et al. 2020 [25]. 
Finalmente, también se constató que el incremento en la proporción de ácidos grasos omega-3 se acompañó de un cambio en el cociente entre ácidos omega-3 y omega-6, que disminuyó desde un valor inicial de 36,2 (sin suplemento de aceite de lino) hasta un valor de 2 (con $4 \%$ de suplemento), un valor que se considera más saludable para la dieta humana.

\section{Conclusión}

La especie presenta un perfil lipídico con predominancia de ácidos grasos poliinsaturados, con capacidad de síntesis de novo de ácidos grasos $\omega-6$ y amplia posibilidad de aumentar los niveles de $\omega$-3 mediante modificaciones en los ácidos grasos de la dieta. Estas constataciones sobre las posibilidades de modificar el perfil lipídico a través de la dieta permiten el desarrollo de múltiples estrategias en cuanto a modificaciones en la dieta para optimizar y adaptar las producciones dependiendo de lo que se buscara. Dada la observada plasticidad de la cantidad de grasa y del perfil lipídico también podríamos plantear su empleo en alimentación humana con diferentes enfoques.

La producción intensiva de estos insectos aún se está optimizando y expandiendo. Prueba de ello es que en los últimos años se ha ido implementando en diversos países nueva legislación para mejorar el control de estas producciones. A nivel nutricional ofrecen muy buenos resultados en cuanto a la cantidad y calidad de su composición proteica, pero como hemos podido ver también ofrece posibilidades de modificación, según interés, del perfil lipídico. La producción de estos animales tiene un interesante potencial en relación con la sostenibilidad de los sistemas alimentarios, prueba de ello es su comparativamente baja huella ecológica y el hecho de posibilitar, más que otras la economía circular, además de sus buenas características a nivel nutricional ya comentadas. La especie que hemos tratado, Acheta domesticus, podría utilizarse para abrir camino, de forma escalada, en la alimentación tanto humana como animal. Las amplias posibilidades a la hora de formular su dieta brindan la oportunidad de realizar modificaciones en la composición final de la harina obtenida, en los porcentajes de proteína, de lípidos y dentro de éstos últimos, los porcentajes de los diferentes ácidos grasos del perfil lipídico.

\section{Referencias}

[1] Ramos-Elorduy, J. (2004). La etnoentomología en la alimentación, la medicina y el reciclaje, p. 329-413. En J. Llorente, J. Morrone, O. Yáñez \& I. Vargas (eds.). Biodiversidad Taxonomía y Biogeografía de Artrópodos de México. Hacia una síntesis de su conocimiento IV. México D.F, CONABIO/UNAM, pp.329-417.

[2] Huis, A., Van Itterbeeck, J., Klunder, H., Mertens, E., Halloran, A., Muir, G. y Vantomme, P. (2013). Edible Insects: Future Prospects For Food And Feed Security. Roma: Food and Agriculture Organization of the United Nations FAO Forestry Paper 171.
[3] Kulma, M., Kouřimská, L., Plachý, V., Božik, M., Adámková, A., \& Vrabec, V. (2019). Effect of sex on the nutritional value of house cricket, Acheta domestica L. Food Chemistry, 272, 267-272M.C.

[4] Collavo, A., Glew, R.H., Huang, Y.S., Chuang, L.T., Bosse, R., \& Paoletti, M.G. (2005). "House cricket small-scale farming", en Paoletti, M. (ed) Ecological Implications of Minilivestock. Potential of Insects, Rodents, Frogs and Snails. Enfield: Science Publishers, pp. 519-545.

[5] Miech, P. (2018). Cricket Farming An Alternative for Producing Food and Feed in Cambodia. Tesis, Department of Animal Nutrition and Management. Swedish University of Agricultural Sciences.

[6] Nissen, L., Samaei, S., Babini, E. and Gianotti, A., 2020. Gluten free sourdough bread enriched with cricket flour for protein fortification: Antioxidant improvement and Volatilome characterization. Food Chemistry, 333, p.127410.

[7] Kouřimská, L. and Adámková, A., 2021. Nutritional and sensory quality of edible insects. NFS Journal, 4 pp 22-26.

[8] Igual, M., García-Segovia, P. and Martínez-Monzó, J., 2020. Effect of Acheta domesticus (house cricket) addition on protein content, colour, texture, and extrusion parameters of extruded products. Journal of Food Engineering, 282, p.110032.

[9] Fernandez-Cassi, X., Supeanu, A., Vaga, M., Jansson, A., Boqvist, S. and Vagsholm, I., 2019. The house cricket (Acheta domesticus) as a novel food: a risk profile. Journal of Insects as Food and Feed, 5(2), pp.137-157.

[10] Teo, L., \& Woodring, J. (1985). Digestive enzymes in the house cricket Acheta domesticus with special reference to amylase. Comparative Biochemistry And Physiology Part A: Physiology, 82(4), 871-877

[11] Chapman, R., Simpson, S., \& Douglas, A. (2013). The insects (pp. 4772). Cambridge: Cambridge University Press.

[12] Blomquist, G., Borgeson, C., \& Vundla, M. (1991). Polyunsaturated fatty acids and eicosanoids in insects. Insect Biochemistry, 21(1), 99106.

[13] Patton, R.L. (1967). Oligidic diets for Acheta domesticus (Orthoptera: Gryllidae). Annals Of The Entomological Society Of America, 60(6), pp.1238-1242.

[14] Dossey, A., Morales-Ramos, J., \& Rojas, M. (2016). Insects As Sustainable Food Ingredients (p. 180). Saint Louis: Elsevier Science \& Technology.

[15] Gasco, L., Biancarosa, I. and Liland, N., 2020. From waste to feed: A review of recent knowledge on insects as producers of protein and fat for animal feeds. Current Opinion in Green and Sustainable Chemistry, 23, pp.67-79.

[16] Paul, A., Frederich, M., Megido, R., Alabi, T., Malik, P., Uyttenbroeck, R., Francis, F., Blecker, C., Haubruge, E., Lognay, G. and Danthine, S. (2017). Insect fatty acids: A comparison of lipids from three Orthopterans and Tenebrio molitor L. larvae. Journal of Asia-Pacific Entomology, 20(2), pp.337-340.

[17] Sánchez-Muros, M., Barroso, F., \& Manzano-Agugliaro, F. (2020). Insect meal as renewable source of food for animal feeding: a review. Journal of Cleaner Production (65), pp.16-27.

[18] Singh, Y., Cullere, M., Kovitvadhi, A., Chundang, P. and Dalle Zotte, A., 2020. Effect of different killing methods on physicochemical traits, nutritional characteristics, in vitro human digestibility and oxidative stability during storage of the house cricket (Acheta domesticus L.). Innovative Food Science \& Emerging Technologies, 65, p.102444.

[19] Bawa, M., Songsermpong, S., Kaewtapee, C., \& Chanput, W. (2020). Effect of Diet on the Growth Performance, Feed Conversion, and Nutrient Content of the House Cricket. Journal Of Insect Science, 20(2).

[20] Stanley-Samuelson, D., Jurenka, R., Cripps, C., Blomquist, G., \& de Renobales, M. (1988). Fatty acids in insects: Composition, metabolism, and biological significance. Archives Of Insect Biochemistry And Physiology, 9(1), 1-33. M.

[21] C. Gómez Candela, L.M. Bermejo López, V. Loria Kohen. (2011). Importance of a balanced omega 6/omega 3 ratio for the maintenance of health. Nutritional recommendations. Nutrición Hospitalaria, 26, pp. 323-329, 10.3305/nh.2011.26.2.5117 
[22] Tzompa-Sosa, D., Yi, L., van Valenberg, H., van Boekel, M., \& Lakemond, C. (2014). Insect lipid profile: aqueous versus organic solvent-based extraction methods. Food Research International (62) pp.1087-1094.

[23] Oonincx, D., van Broekhoven, S., van Huis, A., \& van Loon, J. (2015). Feed conversion, survival and development, and composition of four insect species on diets composed of food by-products. PLOS ONE, 10(12), e0144601.

[24] Moreiras, O., Carbajal, A., Cabrera, L., \& Cuadrado, C. (2001). Tablas de composición de alimentos. Madrid: Ediciones Pirámide, pp. 89-91.

[25] Oonincx, D., Laurent, S., Veenenbos, M., \& Loon, J. (2019). Dietary enrichment of edible insects with omega 3 fatty acids. Insect Science, 27(3), 500-509. 